\title{
Reflectarray Antennas: Analysis and Synthesis Techniques
}

\author{
Sandra Costanzo \\ Dipartimento di Elettronica, Informatica e Sistemistica, Università della Calabria, 87036 Rende, Italy \\ Correspondence should be addressed to Sandra Costanzo, costanzo@deis.unical.it
}

Received 2 September 2012; Accepted 2 September 2012

Copyright ( 2012 Sandra Costanzo. This is an open access article distributed under the Creative Commons Attribution License, which permits unrestricted use, distribution, and reproduction in any medium, provided the original work is properly cited.

The Reflectarray consists of a surface or aperture that is characterized by a surface impedance, and a primary radiator that illuminates this surface The surface impedance can be chosen to produce reflected fields that form a pencil beam in any direction, or shaped beams

(Berry et al. (1963))

The reflectarray concept was firstly introduced in 1963 [1] to define a new class of antennas combining the performance versatility of arrays with the simplicity of reflectors. A waveguide array type was presented in [1] to validate the reflectarray principle, but the real interest in this new kind of antenna came in the late 1980s [2-4], when low-profile reflectarrays were introduced.

Printed reflectarrays consist of an array of microstrip elements illuminated by a primary radiator, typically represented by a horn antenna. The printed elements, giving a flat reflecting surface, are designed to reradiate the incident field with a planar phase front in a prescribed direction. The use of microstrip technology gives significant improvements, in terms of low-cost, less-weight, and easy installation, with respect to standard parabolic reflectors. On the other hand, the feeding mechanism inherited from reflector antennas avoids the disadvantages, in terms of complexity and losses, due to the feeding networks usually adopted in phased arrays.

Various configurations of passive printed reflectarrays, adopting different tuning mechanisms, have been introduced in literature [5-8]. Dynamic phase control techniques have been also investigated for the design of active reflectarrays [9-13], and significant achievements have been obtained in the improvement of bandwidth performances [14-16]. Analysis methods based on different approaches, such as that assuming an infinite array model [17] or that considering each radiator as an isolated element on a grounded dielectric substrate [18], have been considered in literature, and various numerical methods have been implemented for both single-layer and multilayer reflectarrays. A very efficient synthesis technique, based on the intersection approach [19] and properly taking into account both the amplitude and the phase of the field reflected by the reflectarray unit cell, has been developed and applied in [20-22]. Experimental methods alternative to the standard waveguide simulator approach [23] have been developed, with the adoption of open resonator $[24,25]$ and near field [26-28] techniques for the characterization of both passive and active reflectarray unit cell.

Today, the use of microstrip reflectarrays is largely diffused in many application fields, such as remote sensing and satellite communications. With the new emerging technologies, advanced features are required in terms of broadband, dual-polarization, and beam-scanning operations.

The aim of this special issue is to provide an overview of innovative design methodologies and simulation techniques to face the increasing complexity of new microstrip reflectarray configurations.

This special issue collects 13 papers from 36 authors belonging to several countries and institutions. New emerging ideas and results are presented, with particular focus on the following issues: 
(i) synthesis of dual-polarized and conformal reflectarrays;

(ii) reflectarray elements with enhanced bandwidth features;

(iii) beam-scanning and reconfigurable reflectarrays at microwave and millimeter frequencies;

(iv) innovative reflectarrays analysis techniques;

(v) new methods for the numerical and the experimental characterization of reflectarray elements.

The first paper by Venneri et al. proposes a new active reflectarray configuration able to guarantee a full phase tuning range with a single varactor diode and presents interesting results coming from the synthesis of a reconfigurable reflectarray prototype.

The second paper by Venneri et al. presents innovative results concerning the bandwidth evaluation and improvement of slot-coupled reflectarrays.

The paper by Di Massa et al. proposes a new technique for the experimental evaluation of the phase response relative to reflectarray elements, which is based on the adoption of an open cavity system.

The paper by Marnat et al. presents and compares four optimization strategies for the synthesis of dual-polarized microstrip reflectarrays.

The paper by Nayeri et al. introduces a generalized analysis approach to characterize the radiation performance of conformal reflectarrays.

The paper by Tienda et al. presents a general analysis technique for both dual-reflector antennas with a reflectarray subreflector and dual-reflectarray antennas with flat or parabolic main reflectarray.

The paper by Tayebi et al. proposes a new method to automatically design reflectarray antennas.

The paper by Alvarez et al. presents a phase-only optimization method as a systematic framework able to deal with a general reflectarray synthesis problem.

The paper by Van Ha et al. proposes a concentric double square ring configuration as innovative radiating element for large bandwidth reflectarrays.

The paper by Freni et al. presents a modelling technique, based on the use of an artificial neural network, and able to characterize the behaviour of arbitrary reflectarray element structure.

The paper by Delgado et al. proposes a new domain decomposition method for a fast analysis of complex reflectarray structures.

The paper by Carrasco et al. discusses the use of an aperture-coupled reflectarray element for reconfigurablebeam antennas using surface-mounted MEMS.

The paper by Ismail and Inam presents an accurate analysis of different radiating elements structures to be used for the design of both passive and active reflectarrays.

\section{Acknowledgments}

The Lead Editor wishes to thank all authors, who have submitted to this special issue some relevant results coming from their research activities, and the reviewers, whose precious suggestions have strongly contributed to improve the quality of the papers. Finally, a very special acknowledgement is due to the Guest Editors and colleagues, Raphaël Gillard and Manuel Arrebola, for their precious cooperation, and to the Editorial Board of the International Journal of Antennas and Propagation, who has made possible the publication of this special issue.

Sandra Costanzo

\section{References}

[1] D. G. Berry, R. G. Malech, and W. A. Kennedy, "The reflectarray antenna," in IEEE Transactions on Antennas and Propagation, vol. 11, pp. 645-651, 1963.

[2] C. S. Malagisi, "Microstrip disc element reflect array," in Proceedings of the Electronics and Aerospace Systems Convention (EASCON'78), pp. 186-192, 1978.

[3] J. P. Montgomery, "A microstrip reflectarray antenna element," in Proceedings of the Antenna Applications Symposium, Urbana, Ohio, USA, 1978.

[4] J. Huang, "Microstrip reflectarray," in Proceedings of the Antennas and Propagation Society International Symposium Digest, vol. 2, pp. 612-615, Ontario, Canada, 1991.

[5] D. M. Pozar and T. A. Metzler, "Analysis of a reflectarray antenna using microstrip patches of variable size," Electronics Letters, vol. 29, no. 8, pp. 657-658, 1993.

[6] R. E. Munson and H. Haddad, "Microstrip reflectarray for satellite communication and RCS enhancement or reduction," U. S. Patent 4, 684, 952, 1987.

[7] J. Huang and R. J. Pogorzelski, "A ka-band microstrip reflectarray with elements having variable rotation angles," IEEE Transactions on Antennas and Propagation, vol. 46, no. 5, pp. 650-656, 1998.

[8] M. R. Chaharmir, J. Shaker, M. Cuhaci, and A. Sebak, "Reflectarray with variable slots on ground plane," IEE Proceedings, vol. 150, no. 6, pp. 436-439, 2003.

[9] S. V. Hum, M. Okoniewski, and R. J. Davies, "Realizing an electronically tunable reflectarray using varactor diode-tuned elements," IEEE Microwave and Wireless Components Letters, vol. 15, no. 6, pp. 422-424, 2005.

[10] R. Sorrentino, R. Vincenti Gatti, L. Marcaccioli, and B. Mencagli, "Beam steering reflectarrays," in Proceedings of the 4th ESA Workshop on Millimetre Wave Technology and Applications, Espoo, Finland, 2006.

[11] H. Legay, G. Caille, E. Girard et al., "MEMS controlled linearly polarized reflect array elements," in Proceedings of the 15th International Symposium of ANtenna Technology and applied ElectroMagnetics (ANTEM '06), Montreal, Canada, 2006.

[12] F. Venneri, S. Costanzo, G. Di Massa, A. Borgia, P. Corsonello, and M. Salzano, "Design of a reconfigurable reflectarray based on a varactor tuned element," in Proceedings of the 6th European Conference on Antennas and Propagation (EuCAP '12), pp. 2628-2631, 2012.

[13] F. Venneri, S. Costanzo, and G. Di Massa, "Reconfigurable aperture-coupled reflectarray element tuned by single varactor diode," Electronics Letters, vol. 48, no. 2, pp. 68-69, 2012.

[14] J. A. Encinar, "Design of two-layer printed reflectarrays using patches of variable size," IEEE Transactions on Antennas and Propagation, vol. 49, no. 10, pp. 1403-1410, 2001.

[15] S. Costanzo, F. Venneri, and G. Di Massa, "Bandwidth enhancement of aperture-coupled reflectarrays," Electronics Letters, vol. 42, no. 23, pp. 1320-1322, 2006. 
[16] F. Venneri, S. Costanzo, G. Di Massa, and G. Amendola, "Aperture-coupled reflectarrays with enhanced bandwidth features," Journal of Electromagnetic Waves and Applications, vol. 22, no. 11-12, pp. 1527-1537, 2008.

[17] D. M. Pozar, "Microstrip reflectarrays: myths and realities," in Proceedings of the International Symposium on Antennas (JINA '04), pp. 175-179, Nice, France, 2004.

[18] F. Venneri, G. Angiulli, and G. Di Massa, "Design of microstrip reflect array using data from isolated patch analysis," Microwave and Optical Technology Letters, vol. 34, no. 6, pp. 411-414, 2002.

[19] O. M. Bucci, G. Franceschetti, G. Mazzarella, and G. Panariello, "Intersection approach to array pattern synthesis," IEE Proceedings $H$, vol. 137, no. 6, pp. 349-357, 1990.

[20] S. Costanzo, F. Venneri, G. Di Massa, and G. Angiulli, "Synthesis of microstrip reflectarrays as planar scatterers for SAR interferometry," Electronics Letters, vol. 39, no. 3, pp. 266-267, 2003.

[21] F. Venneri, S. Costanzo, G. Di Massa, and G. Angiulli, "An improved synthesis algorithm for reflectarrays design," IEEE Antennas and Wireless Propagation Letters, vol. 4, no. 1, pp. 258-261, 2005.

[22] S. Costanzo, F. Venneri, A. Borgia, I. Venneri, and G. Di Massa, "60 GHz microstrip reflectarray on a benzocyclobutene dielectric substrate," IET Science, Measurement and Technology, vol. 5, no. 4, pp. 134-139, 2011.

[23] F. C. E. Tsai and M. E. Bialkowski, "An equivalent waveguide approach to designing of reflect arrays with the use of variablesize microstrip patches," Microwave and Optical Technology Letters, vol. 34, no. 3, pp. 172-175, 2002.

[24] S. Costanzo, G. Di Massa, and O. H. Moreno, "Improved open resonator technique for dielectric characterization," in Proceedings of the 6th European Conference on Antennas and Propagation (EuCAP '12), pp. 2127-2129, 2012.

[25] G. Di Massa, S. Costanzo, and O. H. Moreno, "Accurate circuit model of open resonator system for dielectric material characterization," Journal of Electromagnetic Waves and Applications, vol. 26, no. 5-6, pp. 783-794, 2012.

[26] S. Costanzo and G. Di Massa, "Far-field reconstruction from phaseless near-field data on a cylindrical helix," Journal of Electromagnetic Waves and Applications, vol. 18, no. 8, pp. 10571071, 2004.

[27] S. Costanzo and G. Di Massa, "Direct far-field computation from bi-polar near-field samples," Journal of Electromagnetic Waves and Applications, vol. 20, no. 9, pp. 1137-1148, 2006.

[28] S. Costanzo and G. Di Massa, "Near-field to far-field transformation with planar spiral scanning," Progress in Electromagnetics Research, vol. 73, pp. 49-59, 2007. 

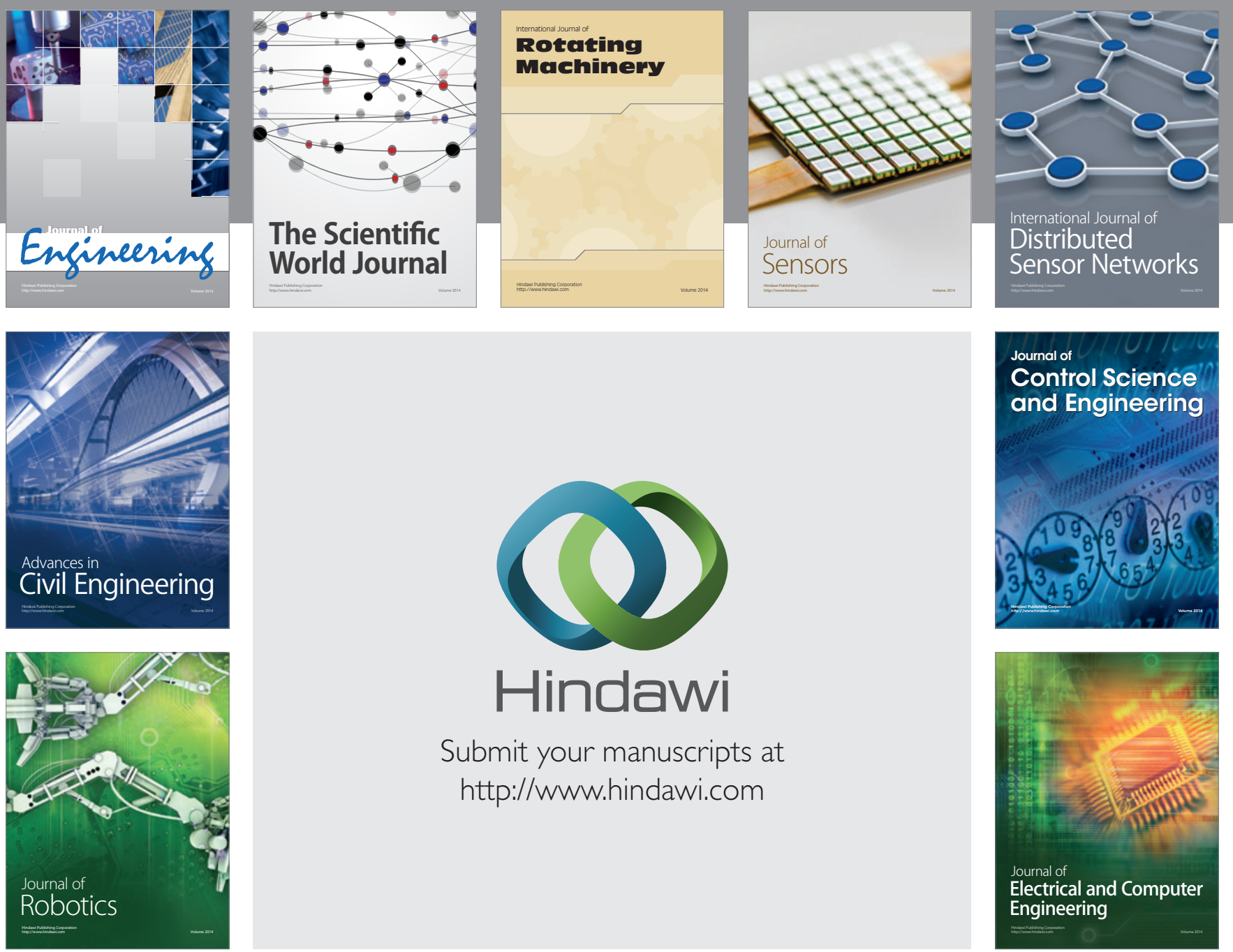

Submit your manuscripts at

http://www.hindawi.com
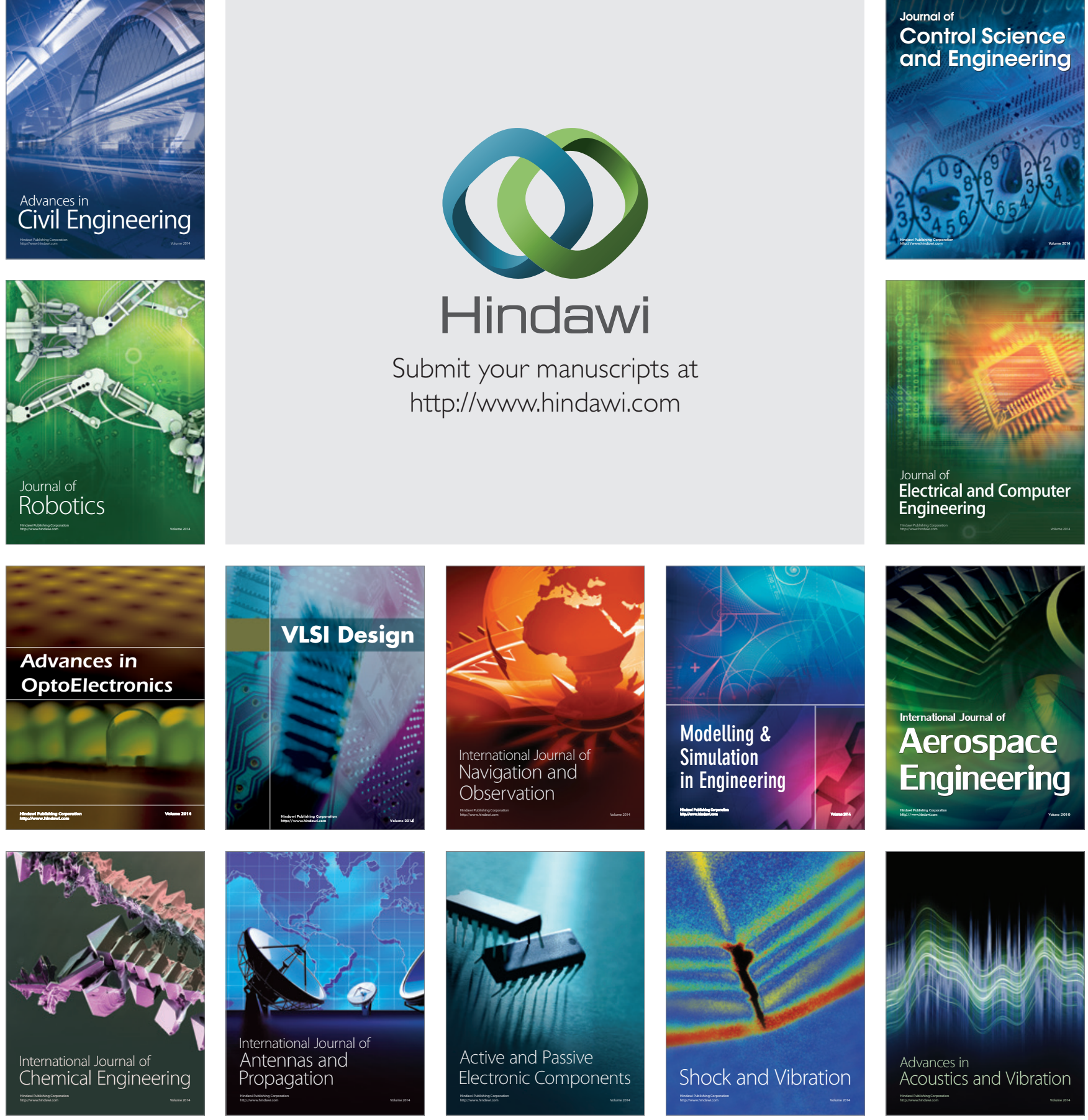